\title{
La production d'eclats dans l'Aurignacien ancien de l'abri Pataud, les Eyzies-de-Tayac, Dordogne
}

\author{
LAURENT CHIOTTI ${ }^{1}$
}

\begin{abstract}
RÉSUMÉ
Les niveaux d'aurignacien ancien de l'abri Pataud (niveaux 14 à 9) ont livré une très forte proportion d'éclats. Même si une part (probablement non négligeable) d'entre eux, sont des déchets issus des productions laminaires ou lamellaires, une grande partie est issue de chaînes opératoires spécifiquement orientées vers la production d'éclats. Etant donné la difficulté pour distinguer l'origine des éclats, nous avons essayé de caractériser les différents types de productions par l'étude des nucléus. Nous avons reconnus dans ces industries différents types de nucléus à éclats: globuleux, informes discoïdes... De plus, à l'occasion de cette étude, nous proposons de définir comme "nucléus à tendance prismatique" des pièces ayant des caractéristiques similaires aux nucléus prismatiques à lames, mais dont la table de débitage, plus courte, a produit des éclats.

Quel que soit le type de production, les éclats sont quasiment toujours en silex local. Ils ont été diversement employés
\end{abstract}

\section{RESUMEN}

Los niveles de auriñaciense antiguo del abri Pataud (niveles 14 a 9) muestran una gran proporción de lascas. Aunque una parte importante de ellas son desechos de la producción de láminas o laminillas, otra parte proviene de cadenas operativas especificamente orientadas hacia la producción de lascas. Vista la dificultad para distinguir el origen de las lascas, hemos intentado caracterizar los diferentes modos de producción a través del estudio de los núcleos. En estas industrias, hemos podido reconocer varios tipos de núcleos para lascas: globulosos, informes, discoides... Además, proponemos definir como núcleos de tendencia prismatica a las piezas que tengan caracteristicas similares a los núcleos prismáticos para obtención de láminas, pero cuya cara de lascado, más corta, a producido lascas.

Sea cual sea el tipo de producción, las lascas son siempre en sílex autóctono y han sido empleadas como soporte de gran número de utensilios. Sin embargo, las

1 USM 103 du Muséum national d'Histoire naturelle, FRE 2676, abri Pataud, 24620. Les Eyzies-deTayac, e-mail: Ichiotti@mnhn.fr 
comme supports de l'outillage, mais ils restent presque toujours majoritaires pour certains types d'outils comme les encoches ou les denticulés.

MOTS-CLEFS

Abri Pataud, Aurignacien ancien, Nucléus, lascas son mayoritarias en las piezas con escotadura o las piezas denticuladas.

PALABRAS CLAVE.

Abri Pataud, Auriñaciense antiguo, Núcleos, Lasca.

Eclat.

\section{INTRODUCTION}

L'abri Pataud, situé dans le village des Eyzies-de-Tayac en Dordogne, a livré une séquence aurignaco-gravettienne composée de 14 niveaux archéologiques. L'Aurignacien y est représenté par les neuf premiers niveaux (niveaux 14 à 6).

Lors de l'étude des industries aurignaciennes (Chiotti, 1999), les niveaux 14 à 11 ont été rattachés à l'Aurignacien ancien ou Aurignacien I. Parmi eux, le niveau 14 , le plus ancien, est daté d'environ 34000 B.P., tandis que le niveau 11 (niveau d'Aurignacien ancien daté, le plus récent) a donné une date d'environ 32000 B.P. (Movius, 1977). Les phases initiales de l'Aurignacien (Aurignacien 0, ou Protoaurignacien) ne sont pas présentes à l'abri Pataud, où la séquence débute par un Aurignacien I classique, comme c'est souvent le cas en Périgord.

Parmi les niveaux d'Aurignacien ancien, les niveaux 9 et 10, ainsi que l'éboulis 10/11 n'ont livré que peu de matériel. En revanche, les niveaux 14 à 11 sont beaucoup plus riches (Tableau I).

Tableau I: Inventaire du matériel lithique des niveaux d'Aurignacien ancien de l'abri Pataud.

\begin{tabular}{ccc}
\hline Niveau & Nb de pièces lithiques & Nb d'outils \\
\hline 9 & 285 & 29 \\
10 & 772 & 74 \\
$10 / 11$ & 279 & 63 \\
11 & 6488 & 1197 \\
12 & 2839 & 515 \\
13 & 1952 & 110 \\
14 & 1466 & 231 \\
\hline
\end{tabular}

II faut signaler que pour les niveaux 13 et 14 , il y a eu des problèmes de conservation du matériel extrait lors de la fouille, à la suite desquels une partie des pièces a perdu son attribution. Ces deux niveaux étaient donc beaucoup plus riches en matériel lithique que ne le laissent entrevoir les décomptes ci-dessus. En 
l'état actuel des collections, les niveaux d'Aurignacien ancien les plus représentatifs sont donc les niveaux 11 et 12 .

\section{QUANTIFICATION DES ECLATS ET IMPLICATIONS}

Tous les niveaux d'Aurignacien ancien ont livré des proportions d'éclats importantes (Tableau II) qui varient d'un minimum de près de $75 \%$ (niveau 12) à plus de $92 \%$ (niveau 9 ). Seul le niveau 14 présente un taux d'éclats assez faible, avec moins de $65 \%$, mais il faut rappeler qu'une part relativement importante du matériel de ce niveau est absente.

Tableau II. Répartition des types de supports dans les différents niveaux d'Aurignacien ancien de l'abri Pataud.

\begin{tabular}{cccccc}
\hline \multirow{2}{*}{ Niveau } & & éclats & lames & Lamelles & Total \\
\hline \multirow{2}{*}{9} & $\mathrm{Nb}$ & 253 & 18 & 3 & 274 \\
& $\%$ & 92,34 & 6,57 & 1,09 & 100,00 \\
\hline \multirow{2}{*}{10} & $\mathrm{Nb}$ & 631 & 107 & 21 & 759 \\
& $\%$ & 83,14 & 14,10 & 2,77 & 100,00 \\
\hline \multirow{2}{*}{$10 / 11$} & $\mathrm{Nb}$ & 238 & 28 & 7 & 273 \\
& $\%$ & 87,18 & 10,26 & 2,56 & 100,00 \\
\hline \multirow{2}{*}{11} & $\mathrm{Nb}$ & 5179 & 1098 & 211 & 6488 \\
& $\%$ & 79,82 & 16,92 & 3,25 & 100,00 \\
\hline \multirow{2}{*}{12} & $\mathrm{Nb}$ & 2048 & 417 & 274 & 2739 \\
& $\%$ & 74,77 & 15,22 & 10,00 & 100,00 \\
\hline \multirow{2}{*}{13} & $\mathrm{Nb}$ & 1523 & 196 & 145 & 1864 \\
& $\%$ & 81,71 & 10,52 & 7,78 & 100,00 \\
\hline \multirow{2}{*}{14} & $\mathrm{Nb}$ & 879 & 307 & 172 & 1358 \\
& $\%$ & 64,73 & 22,61 & 12,67 & 100,00 \\
\hline
\end{tabular}

Ces éclats ont deux origines possibles. Soit ils sont le produit de certaines étapes des chaînes opératoires laminaires ou lamellaires, comme la préparation ou l'entretien des nucléus, et sont alors des déchets. Soit ils proviennent de débitages spécifiques et sont alors les produits recherchés. La plupart du temps, il est très difficile, de différencier ces deux types d'éclats. Cependant, dans l'Aurignacien ancien de l'abri Pataud, il apparaît clairement qu'une proportion importante des éclats a été produite volontairement, et ce pour deux raisons. D'une part, les débitages laminaires in situ (générateurs de déchets) semblent relativement peu nombreux, et d'autre part, nous avons retrouvé une quantité importante de nucléus à éclats (Tableau IV). 
Tableau III. Pourcentage d'éclats et indice laminaire dans l'outillage des différents niveaux d'Aurignacien ancien de l'abri Pataud.

\begin{tabular}{cccc}
\hline Niveau & Nb d'outils & pourcentage d'éclats & indice laminaire \\
\hline $\mathbf{9}$ & 29 & 61,54 & 38,46 \\
$\mathbf{1 0}$ & 74 & 59,65 & 40,35 \\
$\mathbf{1 0 / 1 1}$ & 63 & 65,26 & 34,74 \\
$\mathbf{1 1}$ & 1197 & 43,68 & 56,32 \\
$\mathbf{1 2}$ & 515 & 54,17 & 45,83 \\
$\mathbf{1 3}$ & 110 & 47,44 & 52,56 \\
$\mathbf{1 4}$ & 231 & 27,22 & 72,78 \\
\hline
\end{tabular}

La proportion d'éclats dans l'outillage des niveaux d'Aurignacien ancien est très variable (Tableau III). A l'exception du niveau 14 dont l'outillage ne présente que $27,22 \%$ d'éclats $^{2}$, elle varie d'un minimum de $43,68 \%$ (niveau 11 ) jusqu'à un maximum de $65,26 \%$ (éboulis 10/11). Dans la plupart des niveaux, les éclats ont donc été très fortement utilisés comme support de l'outillage.

La seule industrie qui présente un indice laminaire de l'outillage élevé (ILO = 72,78 ), habituel pour cette période (Demars, 1994), est celle du niveau 14. Après le niveau 14, c'est le niveau 11 qui présente le plus d'outils sur lame, mais dans des proportions bien moins importantes (ILO $=56,32$ ). Dans tous les autres niveaux l'indice laminaire est très bas (Tableau III).

Ces valeurs anormalement basses de l'indice laminaire pour l'Aurignacien ancien peuvent trouver une explication dans des variations du mode d'occupation du site au cours de cette période. En effet, lors de l'étude de l'ensemble des industries aurignaciennes du site de l'abri Pataud (Chiotti, 1999), nous avions noté que les niveaux 14 à 9 correspondent tous à de l'Aurignacien ancien, mais avec des modes d'occupation différents.

Deux modes d'occupation avaient pu être identifiés:

- des occupations brèves, correspondant certainement à des haltes de chasse très temporaires (niveaux 10 et 13 , éboulis 10/11);

- des occupations de plus longue durée, avec des quantités d'artefacts plus importantes, ainsi que la présence de foyers aménagés (niveaux 14, 12 et 11).

Le niveau 9 semble quant à lui n'être que la partie latérale d'une occupation plus importante. Une étude détaillée du niveau 11 (Chiotti et al., 2003) nous a

${ }^{2}$ Le problème de la perte d'attribution d'une partie du matériel lithique des niveaux 14 et 13 ne se pose pas pour l'outillage. En effet l'outillage étant marqué, il n'a pas subi les mêmes désagréments que les produits de débitage bruts non marqués. 
permis de déterminer que ce dernier ne correspond pas à une occupation de longue durée, mais plutôt à une succession d'occupations relativement brèves.

En corrélant ces données avec l'indice laminaire, les faits suivants apparaissent:

- Le niveau 14 correspond à une occupation importante, avec un outillage bien développé, d'indice laminaire élevé, situation tout à fait classique pour l'Aurignacien ancien en Périgord.

- Les niveaux 10 et 13 ainsi que l'éboulis $10 / 11$ sont des haltes de courte durée dont les industries présentent un indice laminaire bas. Cela correspond au fait que durant de telles haltes, les hommes n'ont pas développé des activités de taille importantes, mais ont plutôt débité des éclats pour un usage immédiat. Les débitages laminaires in situ sont alors limités, voire inexistants.

- Le niveau 11 correspond quant à lui à une série de haltes de chasse. Son industrie présente un indice laminaire faible pour un Aurignacien ancien, mais relativement plus élevé que celui des niveaux précédents. Cela pourrait provenir du fait que suite à la répétition de passages dans l'abri, un nombre un peu plus important d'outils élaborés sur lames y ait été abandonné.

- Etant donné son indice laminaire très bas, le niveau 12 , que nous avions considéré dans un premier temps comme une occupation d'assez longue durée pourrait s'avérer correspondre, comme le niveau 11, à une succession de passages dans l'abri.

En résumé, lors de brefs passages dans l'abri, les hommes ne fabriquent pas une grande quantité d'outils sur place. On trouve souvent des pièces laminaires isolées qui n'ont pas été débitées sur le site, la grande majorité des débitages effectués sur place étant orientés vers la production d'éclats.

Au contraire, lors des séjours plus importants, comme celui du niveau 14 un plus grand nombre d'outils sont fabriqués sur place, avec une variété plus importante. C'est donc là que l'on trouve les outillages "les plus complets", ainsi qu'un débitage de lames in situ plus important que dans les autres niveaux. Le débitage d'éclats reste néanmoins très présent dans ces niveaux.

En définitive, il apparaît que dans la plupart des niveaux d'Aurignacien ancien de l'abri Pataud, les débitages effectués dans l'abri sont majoritairement orientés vers une production d'éclats.

\section{LES PRODUCTIONS D'ECLATS}

Dans tous les niveaux d'Aurignacien ancien, différentes techniques ont été employées pour l'obtention d'éclats. Nous les avons identifié essentiellement sur la base de l'étude des nucléus. 


\subsection{Les nucléus globuleux et les nucléus informes}

\subsubsection{Définition}

Nous avons regroupé les nucléus globuleux et les nucléus informes du fait de leur mode opératoire très similaire. Ces deux types de nucléus ont été définis de la façon suivante.

- Les nucléus globuleux sont des pièces dont le débitage est conduit en exploitant tour à tour toutes les faces d'un bloc, les surfaces d'enlèvement devenant ensuite plan de frappe. Denise de Sonneville Bordes (SonnevilleBordes, 1960: 20) les définit comme des «nucléus à éclats présentant grossièrement une forme polyédrique, les éclats ayant été obtenus en choisissant successivement comme plan de frappe les faces d'enlèvement d'éclats précédents, et en faisant tourner le nucléus à peu près régulièrement entre les mains". André Leroi-Gourhan (Leroi-Gourhan, 1964: 9) dénommait également ces pièces nucléus polyédriques.

- Les nucléus informes présentent un type de débitage assez similaire qui pour plusieurs auteurs est un dérivé de celui des nucléus globuleux. Georges Laplace les définit comme un «type dégradé du nucléus globuleux» (Laplace, 1964: 19). Denise de Sonneville-Bordes (Sonneville-Bordes, 1960: 20) les définit comme des "nucléus à éclats ne présentant aucune forme déterminée, d'où les éclats ont été obtenus de la même façon [que ceux du nucléus globuleux] mais sans que les enlèvements soient faits régulièrement»".

J. Tixier regroupe ces deux débitages sous le terme de débitage sans prédétermination (Tixier et al., 1980: 42) ou de débitage peu élaboré (Inizan et al., 1995: 61), et les définit comme suit: «Le tailleur choisit chaque fois un endroit où frapper en tenant compte de la morphologie de son nucléus [...]. Les nucléus n'ont dans ce cas pas de plan de frappe préférentiel, ils tendent généralement, si le débitage est assez avancé, vers des formes globuleuses. "

Les nucléus globuleux et informes résultent donc un même type de débitage mené avec plus ou moins de régularité sur le bloc. Ils constituent souvent la majorité des effectifs des nucléus à éclats dans les industries aurignaciennes (Perpère, Schmider, 2002; Sonneville-Bordes, 1960, 1970...)

\subsubsection{Les pièces de l'Aurignacien ancien de l'abri Pataud}

Ce type de débitage est bien représenté dans l'Aurignacien ancien de l'abri Pataud, avec une quarantaine de nucléus (4 globuleux et 36 informes; Fig. 1a et b). Il est surtout très présent dans les deux niveaux les plus importants: le niveau 11 avec 20 nucléus et le niveau 14 avec 12 nucléus (Tableau IV). 
La production d'eclats dans l'Aurignacien ancien de l'abri Pataud, Les Eyzies-de-Tayac...

Tableau iV. Types de nucléus dans les différents niveaux d'Aurignacien ancien de l'abri Pataud.

\begin{tabular}{|c|c|c|c|c|c|c|c|}
\hline $\begin{array}{l}\text { Niveau } \\
\text { Type nucléus }\end{array}$ & 9 & 10 & $10 / 11$ & 11 & 12 & 13 & 14 \\
\hline Globuleux & & & & 4 & 1 & & 4 \\
\hline Informe & 3 & 3 & 1 & 14 & 6 & 1 & 8 \\
\hline Sur arête & & & & 2 & 1 & & \\
\hline Discoïde & & & & 5 & & & 2 \\
\hline Levallois & & & & 2 & & & \\
\hline Tendance prismatique & & 6 & 1 & 50 & 7 & 1 & 16 \\
\hline Sur éclat & & & & 1 & & 1 & 1 \\
\hline Composite & & & & 2 & & & \\
\hline Prismatique repris pour éclats & & & & 1 & 1 & 1 & 3 \\
\hline Prismatique & & 1 & 1 & 6 & 7 & & 7 \\
\hline
\end{tabular}

Dans les niveaux 11 et 12, nous avons également identifié quatre pièces (trois dans le niveau 11 et une dans le niveau 12) qui constituent une variante de ce type de débitage. II s'agit d'un débitage d'éclats alterné de part et d'autre d'une arête (Fig. 1c). Nous considérons qu'il s'agit d'une variante des nucléus globuleux ou
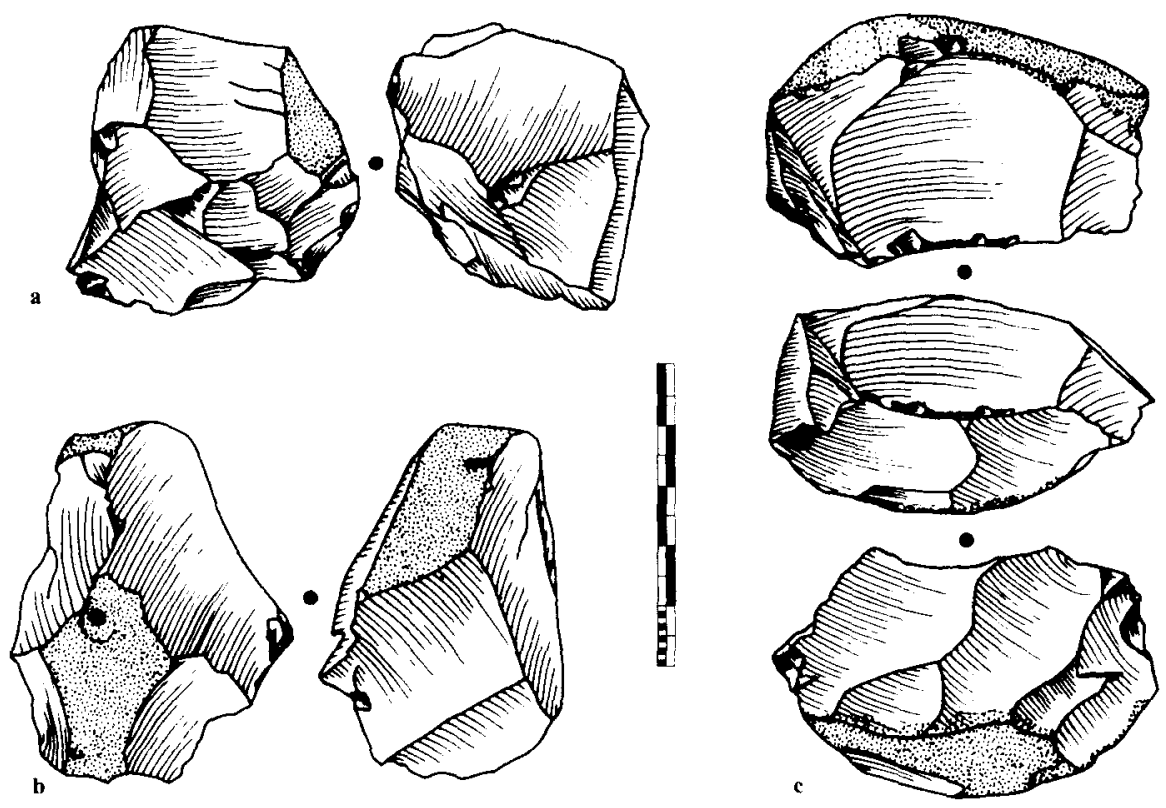

Fig. 1. Nucléus à éclats de l'Aurignacien ancien de l'abri Pataud (niveau 11) (dessins L. Chiotti). $a$ : nucléus globuleux; $b$ : nucléus informe; $c$ : débitage d'éclats sur une arête. 
informes car la technique est similaire, c'est à dire que chaque nouvel enlèvement utilise comme plan de frappe le négatif d'un éclat précédent, sans qu'il y ait au préalable de préparation particulière.

Ces débitages s'apparentent fortement à la réalisation d'une crête, cependant après l'analyse de chacune de ces pièces, nous pensons qu'il s'agit vraiment de débitages d'éclats. Les deux premières pièces sont des nucléus de petites dimensions qui ne se prêtent pas à un débitage élaboré (en particulier laminaire), et donc sur lesquels il n'y avait pas de raison de réaliser une crête. La troisième pièce est un nucléus plus gros, mais dont la matière première de mauvaise qualité ne se prête pas non plus à un débitage élaboré. La quatrième pièce est un nucléus qui présente deux types de débitages différents sur chacune de ses faces: un débitage à tendance prismatique (cf. infra) d'un côté et un débitage sur arête de l'autre. Cette pièce est celle qui fait le plus penser à un crête, le bloc étant de dimensions suffisantes et la matière de bonne qualité. Cependant, si le but avait été la réalisation d'une crête, celle-ci aurait probablement été débitée, d'autant plus n'y a aucune raison apparente empêchant son utilisation en tant que tel.

\subsection{Les nucléus discoïdes}

\subsubsection{Définition}

Le débitage discoïde a été défini par $F$. Bordes (Bordes, 1950: 22) pour le Paléolithique ancien et moyen, sous le nom de débitage moustérien. Le nucléus discoïde est un nucléus sur lequel les éclats sont détachés alternativement sur les deux faces et qui tend à prendre la forme de deux pyramides opposées par la base.

Pour le Paléolithique supérieur, André Cheynier (Cheynier, 1949: 52) les décrit dans le Solutréen de Badegoule, sous le nom de nuclei-disques, comme "des nucléus constitués par deux faces convexes réunies par une arête toujours incomplète circulaire, quelque peu sinueuse». Denise de Sonneville-Bordes (Sonneville-Bordes, 1960: 20) quand à elle définit le nucléus discoïde comme "un nucléus à éclats plats, avec des enlèvements centripètes analogues à ceux du Moustérien». Selon J. Tixier (Tixier et al., 1980: 43), "ils ont une silhouette généralement circulaire, une section biconvexe dissymétrique: la face la moins bombée est celle formée par les négatifs d'enlèvement des éclats, l'autre [...étant...] formée par les négatifs de préparation du plan de frappe". Plus récemment, Eric Boëda a défini ce qu'il appelle la conception discoïde du débitage, par l'interaction d'une série de critères techniques (Boëda, 1993). II s'agit d'une conception volumétrique du débitage qui permet de prédéterminer la forme de certains produits.

Lorsque les deux faces du nucléus sont formées par des enlèvements redressés, il est parfois nommé nucléus bipyramidal (Leroi-Gourhan, 1964: 9). 
Pour J. Tixier (Tixier, 1960: 170), cela se produit lorsqu' “il n'est plus possible de distinguer le plan de frappe et la table d'enlèvement, car cette dernière est pyramidale».

\subsubsection{Les pièces de l'Aurignacien ancien de l'abri Pataud.}

Les nucléus discoïdes ne sont représentés que dans deux niveaux par sept pièces: cinq dans le niveau 11 et 2 dans le niveau 14 (Tableau IV). II s'agit généralement de pièces de petites dimensions, plus ou moins caractéristiques de ce type de débitage.

Parmi ces nucléus, quatre sont bien caractéristiques de ce type de débitage. Ce sont des pièces de petites dimensions, avec un débitage d'éclats centripètes sur tout le pourtour, et sur les deux faces, la jonction de ces faces formant une arête circulaire. L'un d'entre eux, qui présente des enlèvements assez redressé peut entrer dans la catégorie des nucléus bipyramidaux (Fig. 2a).

Les trois autres nucléus sont plus volumineux et moins caractéristiques. Le premier n'est pas débité sur la totalité du pourtour, essentiellement en raison d'un résidu cortical qui n'a pu être éliminé. Le second n'est débité que sur une seule face, l'autre étant une surface irrégulière, très plate. Le troisième est un petit bloc dont quelques éclats ont été détachés sur les deux faces selon la technique du nucléus discoïde, mais qui reste encore très fortement cortical.
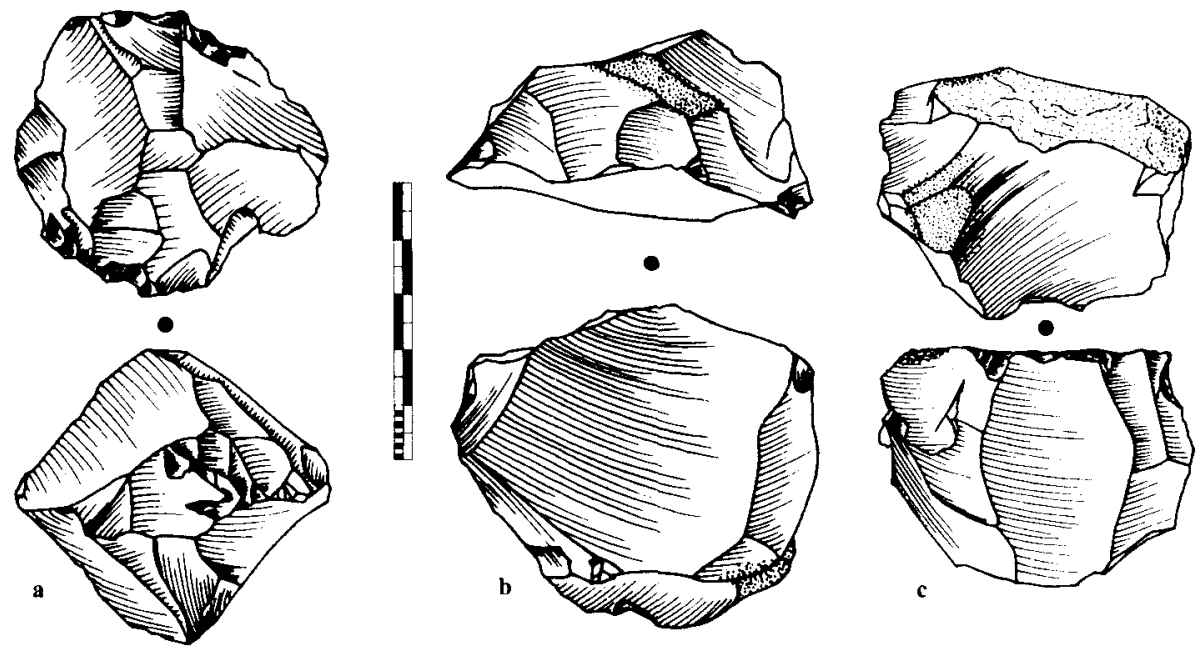

Fig. 2. Nucléus à éclats de l'Aurignacien ancien de l'abri Pataud (dessins L. Chiotti). a: nucléus discoïde (bipyramidal; niveau 11); $b$ : nucléus "de type Levallois" (niveau 11); c: nucléus à tendance prismatique (niveau 12). 
Contrairement à d'autres industries de la même période (Brassempouy, Landes), où les nucléus d'aspect discoïde ont été considérés comme une variante d'un autre type de débitage dénommé débitage trifacial (Bon 1996: 447), il semble bien que nous soyons ici en présence d'un vrai débitage discoïde, même si cela ne concerne que très peu de pièces.

\subsection{Les nucléus "de type levallois»}

La technique levallois, qui se développe au Paléolithique inférieur et moyen est généralement absente au Paléolithique supérieur, à l'exception de cas bien particuliers, comme par exemple pour le débitage de lames de certains sites de la région de Bergerac (Guichard et al., 1989).

Trois nucléus à éclats du niveau 11 présentent des caractères qui évoquent la technique levallois. Le premier est réalisé sur un bloc plat de silex sénonien blond de petites dimensions. Ce bloc présente des aménagements sur son pourtour qui font penser à un aménagement de type levallois. Un éclat a été détaché sur la face supérieure du bloc, mais il ne s'est pas étendu sur toute la face, probablement du fait de la mauvaise qualité du silex. Cet éclat était en partie cortical. Le second nucléus, est réalisé sur un petit bloc de silex sénonien noir qui présente des aménagements sur son pourtour et sur lequel a été détaché un éclat couvrant quasiment toute la surface du nucléus (Fig. $2 b$ ). Enfin, la troisième pièce est un nucléus «composite», qui présente un débitage prismatique sur l'une de ses face et un aménagement de type levallois sur la face opposée.

Dans tous les cas, il s'agit de nucléus qui évoquent la technique levallois, mais qui ne sont pas très caractéristiques. Ces pièces ne nous paraissent pas correspondre à un véritable débitage levallois, qui implique une gestion complexe du volume des blocs de façon à prédéterminer la forme de l'éclat (Boëda, 1994), mais simplement à une volonté d'obtenir un éclat relativement plat à partir de petits blocs.

\subsection{Les nucléus à tendance prismatique}

\subsubsection{Définition}

Les pièces que nous avons dénommé nucléus à tendance prismatique sont des nucléus qui présentent la même organisation qu'un nucléus prismatique classique à lames, mais dont la table de débitage est généralement courte et n'a donné que des éclats, ou parfois des éclats allongés (Chiotti, 1999). 


\subsubsection{Les pièces de l'Aurignacien ancien de l'abri Pataud}

Ce type de nucléus est à l'origine de la plus grande partie de la production d'éclats de l'Aurignacien ancien de l'abri Pataud. II est représenté dans presque tous les niveaux, à l'exception du niveau 9 (Tableau IV). Nous avons dénombré 81 nucléus, dont la plupart se trouvent dans le niveau 11 (50 pièces) et dans le niveau 14 (16 pièces).

Parmi ces nucléus, nous avons distingué quatre catégories: des nucléus à un plan de frappe, à deux plans de frappe, à trois plans de frappe et des nucléus préparés pour être de vrais prismatiques, mais dont le débitage a échoué.

- Les nucléus à un plan de frappe sont les plus nombreux avec 55 pièces (Fig. 2c). La majeure partie d'entre eux sont d'assez petites dimensions, avec une table de débitage ne couvrant qu'une faible partie de la surface du bloc.

- Les nucléus à deux plans de frappe sont représentés par 23 pièces. Selon les cas, les plans de frappe peuvent être opposés ( 9 pièces), orthogonaux (6 pièces) ou croisés (8 pièces). Dans le cas de plans de frappe opposés, les deux plans peuvent agir soit sur la même surface de débitage, soit sur deux surfaces de débitage opposées sur le bloc. Dans le cas de plans de frappe orthogonaux ou croisés, les deux plans agissent toujours sur deux surfaces indépendantes l'une de l'autre, qui peuvent elles mêmes être orthogonales ou croisées.

- Trois nucléus (deux du niveau 14 et un du niveau 11) présentent trois plans de frappe. Ces pièces ont un aspect relativement sphérique du fait du grand nombre de surfaces qui les composent ( 3 plans de frappe et 3 tables de débitage).

- Enfin, certaines pièces que nous avons classé dans les nucléus à tendance prismatique sont en fait des nucléus qui ont été préparés dans l'intention de débiter des lames, mais pour lesquels le débitage laminaire a échoué. Ce cas de figure a pu être identifié pour deux nucléus à 2 plans de frappe et trois nucléus à un plan de frappe, provenant tous du niveau 14.

\subsubsection{Remontage d'un nucléus à tendance prismatique}

Le débitage à tendance prismatique a pu être illustré par le remontage d'un bloc de silex sénonien gris de dimensions assez importantes pour ce type de matériau.

Celui-ci comprend un nucléus, qui dans son état d'abandon est un nucléus à tendance prismatique à un plan de frappe présentant des négatifs d'éclats sur deux faces opposées: des négatifs de petits éclats sur une face et le négatif d'un gros éclat qui couvre toute la face sur l'autre. Le nucléus qui est fracturé, était certainement de dimensions beaucoup plus importantes (Fig. 3a). 

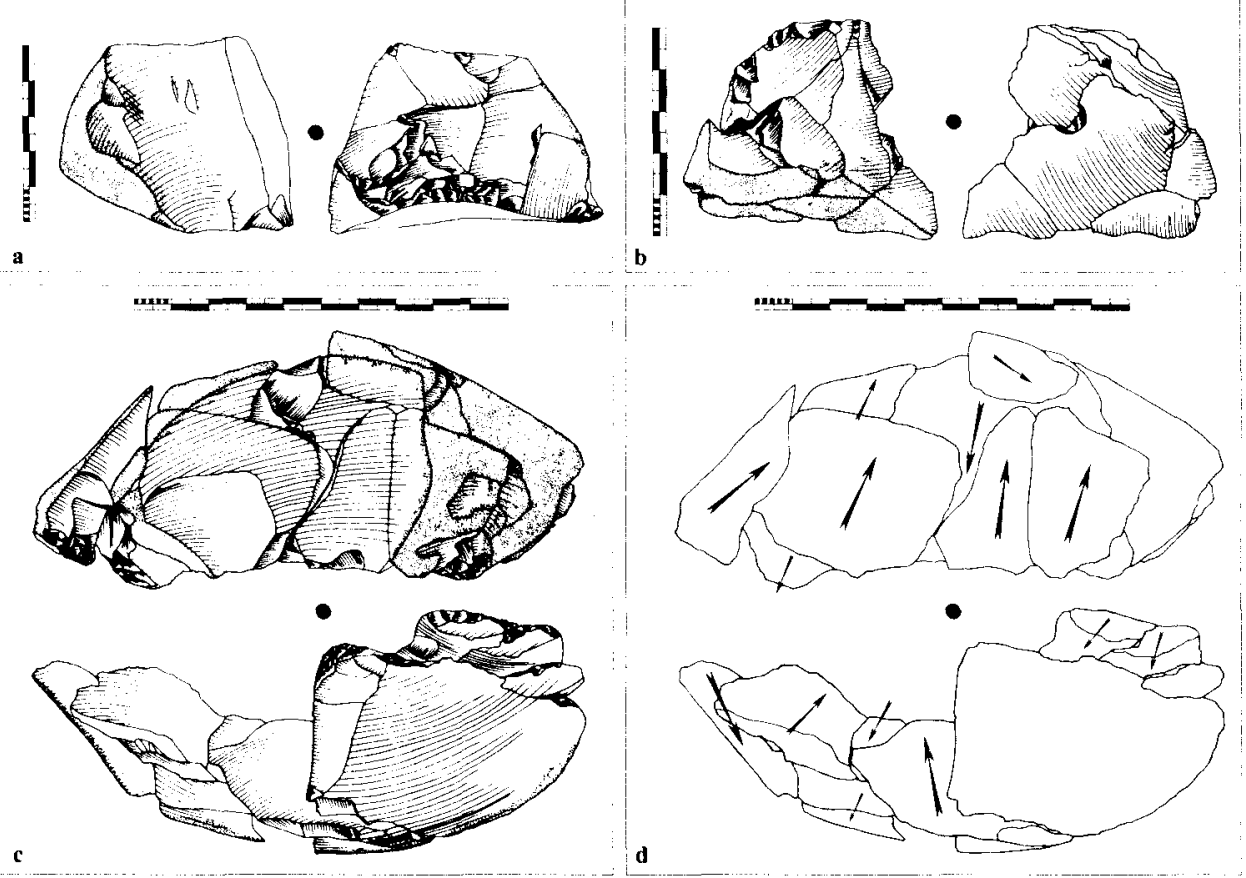

Fig. 3. Remontage d'un nucléus à tendance prismatique du niveau 11 de l'abri Pataud (dessins L. Chiotti). $a$ : nucléus; $b$ : groupe d'éclats non remontés; $c$ et d: remontage.

Le remontage montre que la face qui présente plusieurs négatifs n'a produit que quelques petits éclats (tout au moins pour la moitié du nucléus qui a été retrouvée). En revanche la majeure partie du débitage se développe sur la face qui présente le gros négatif d'éclat.

Sur celle-ci, seuls trois gros éclats ont été détachés dans le même sens que le négatif, à la fin du débitage. Précédemment, une grande série d'éclats avait été débitée dans le sens opposé au négatif présent sur le nucléus (Fig. $3 c$ et d).

Une série d'éclats, non remontés, provenant du même bloc, a probablement été débitée sur la première face, du côté de la moitié non retrouvée du nucléus (Fig. 3b).

Ce remontage illustre bien le principal problème de l'étude des nucléus, dont nous sommes tout à fait conscient ici, à savoir que ceux-ci ne témoignent que des dernières phases du débitage et occultent souvent une part importante de celui-ci. Dans le cas présent, la séquence de débitage d'éclats la plus importante (éclats débités dans le sens opposé au grand négatif d'éclat) n'a laissé aucune trace sur le nucléus. 


\subsection{Les nucléus sur éclat}

Les nucléus sur éclats sont peu nombreux dans l'Aurignacien ancien: trois nucléus et deux gros éclats remontés. Ils sont de plusieurs types.

- Deux d'entre eux (provenant des niveaux 11 et 14) correspondent à de gros éclats épais qui sont utilisés comme nucléus dans leur épaisseur, les produits étant détachés sur le pourtour de l'éclat-nucléus. Le débitage effectué sur ces éclats s'apparente au débitage des nucléus à tendance prismatique. La différence principale étant que les produits sont détachés sur une grande partie du pourtour de l'éclat (Fig. 4). Certains nucléus à tendance prismatique réalisés sur des blocs plats ont d'ailleurs un aspect assez similaire à ces nucléus sur éclats.

Ces quelques pièces peuvent poser problème quant à leur attribution comme nucléus. En effet, étant donné leur forme, il est également possible qu'il s'agisse de préformes de grattoirs carénés (Bon, 2002) qui n'auraient pas été exploitées par la suite. Dans ce cas, ce qui serait recherché, ne serait pas les petits éclats détachés du pourtour des pièces, mais une mise en forme de celles-ci.

- Le niveau 9 a fourni deux éclats plats, de grandes dimensions, qui ont été utilisés comme nucléus (Fig. $5 a$ et $b$ ). Le type de débitage est le même que pour les pièces précédentes, mais ici, ce sont incontestablement des nucléus.

La reconstitution par remontage de ces deux éclats-nucléus (qui remontent entre eux), nous a permis de déterminer qu'ils ont été débités successivement dans un même bloc de silex sénonien (Fig. $5 \mathrm{c}$ ). Ces éclats ont été utilisés dans leur épaisseur pour fournir des éclats de petites dimensions. Pour l'un d'entre eux, la pièce finale a été transformée en un perçoir atypique (Fig. 5d).

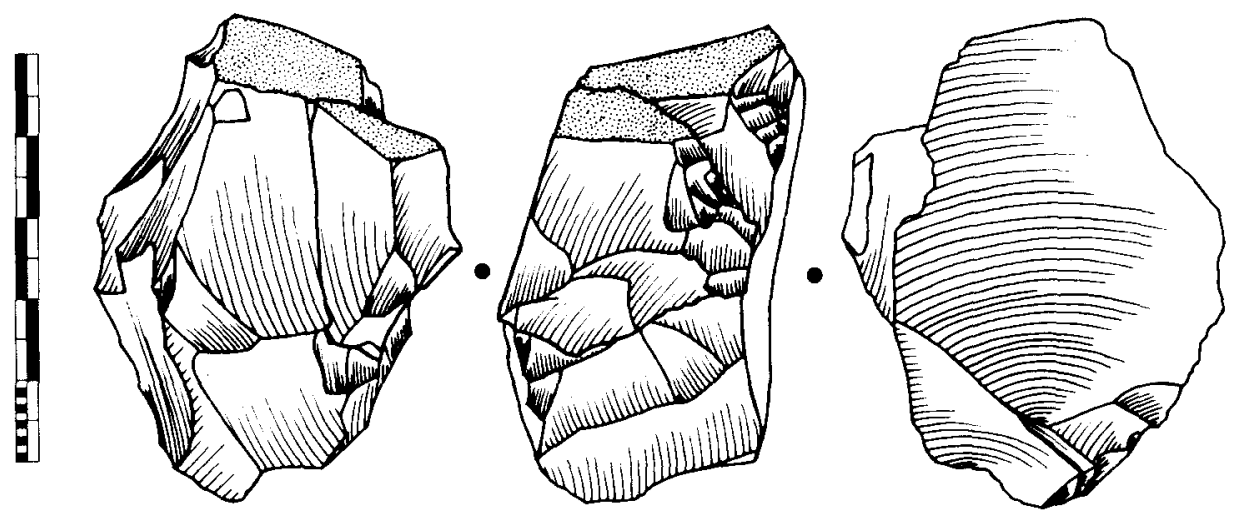

Fig. 4. Nucléus à éclats de l'Aurignacien ancien de l'abri Pataud (dessin L. Chiotti). nucléus sur éclat. 

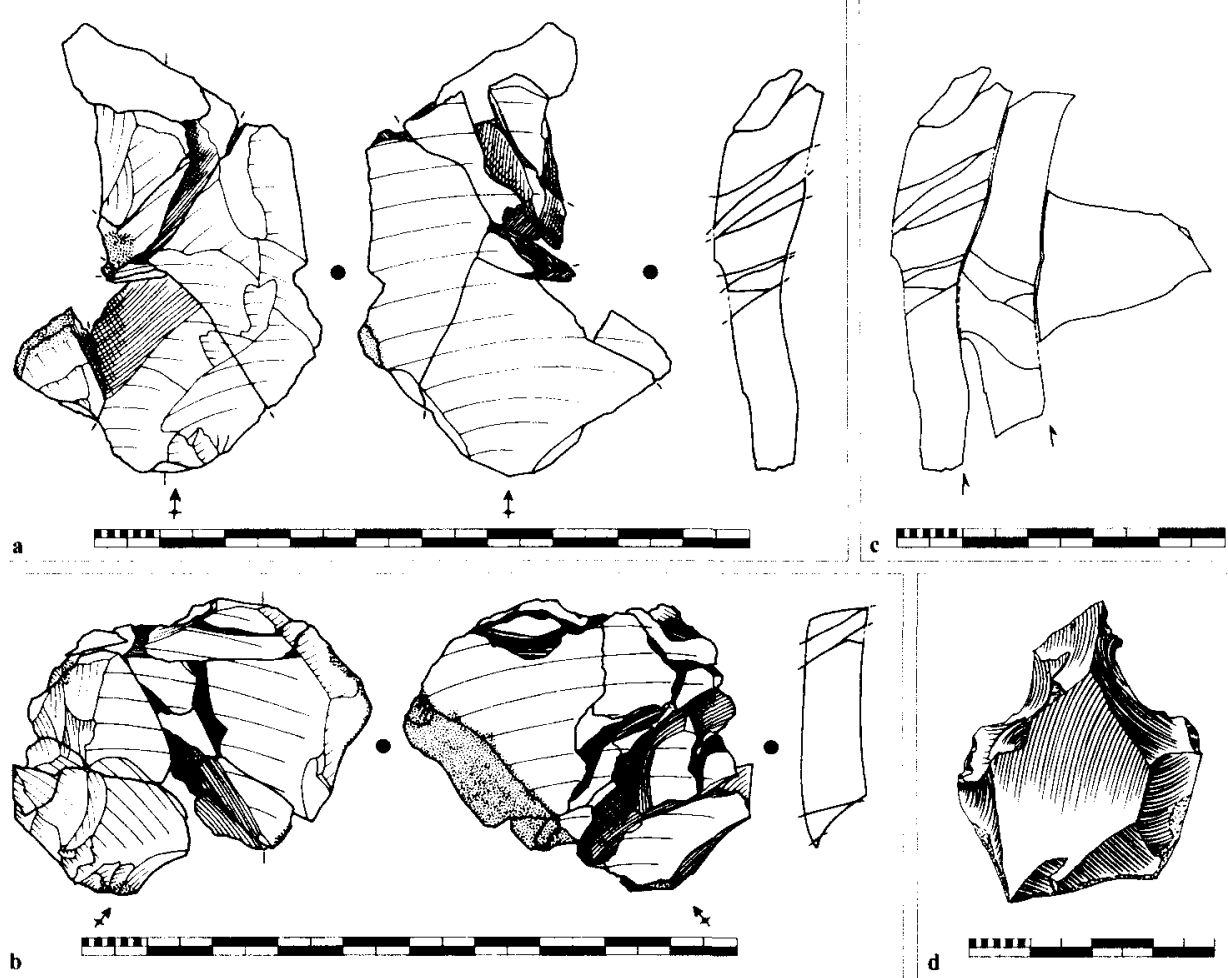

Fig. 5. remontage de nucléus sur éclat du niveau 9 de l'abri Pataud (dessins L. Chiotti. a,b,c; M. Dauvois. d). a: Remontage du premier éclat; $b$ : remontage du second éclat; $c$ : remontage des deux éclats et de leur nucléus vu en coupe; $d$ : perçoir atypique issu du second éclat.

- Enfin, le niveau 13 a fourni un nucléus sur éclat dont la nature est différente. II ne s'agit plus d'un débitage dans l'épaisseur de l'éclat, mais dans sa largeur, qui n'a donné que quelques éclats de très petites dimensions.

\subsection{Nucléus prismatiques repris pour la production d'éclats}

La technique consistant à réutiliser des nucléus ayant servi dans un premier temps à produire des lames et des lamelles pour obtenir des éclats est un procédé qui semble relativement courant dans les industries aurignaciennes. Dans la plupart des cas, il n'y a pas de continuité avec le débitage laminaire précédent, mais une rupture qui se traduit par l'exploitation des différentes surfaces telles que les plans de frappe, l'ancienne table de débitage ou l'arrière du nucléus (Bon 2002: 104 et 135; Bon, Bodu, 2002: 126).

Certains blocs de silex des niveaux d'Aurignacien ancien de l'abri Pataud ont présenté successivement un débitage laminaire (prismatique), puis un débitage d'éclats. 
Il est probable qu'il en soit de même pour certains nucléus à tendance prismatique (peut-être une partie relativement importante d'entre eux?). II s'agirait alors de cas où la seconde phase (débitage d'éclats) aurait effacé toutes traces du débitage laminaire antérieur.

Quelques nucléus ont conservé les traces des deux types de débitages successifs. Ils sont peu nombreux, avec seulement 6 pièces, dont 3 dans le niveau 14 (Tableau IV). Parmi ces pièces, nous avons rencontré plusieurs cas de figure.

- Trois d'entre eux sont des nucléus prismatiques qui ont subi un ravivage du plan de frappe conséquent, ce qui a eu pour effet de réduire la longueur de la table de débitage dans des proportions importantes. A la suite de ce raccourcissement, le nucléus ne donne plus des lames, mais des éclats.

- Dans d'autres cas, l'obtention d'éclats peut avoir lieu sur le fond du nucléus prismatique (1 nucléus), ou encore sur sa face arrière (2 nucléus).

Tous les nucléus de cette catégorie, n'ont fourni que très peu d'éclats, puisque qu'ils portent encore les traces de la première phase laminaire. Cette remarque rend encore plus probable l'hypothèse selon laquelle une partie des nucléus à tendance prismatique serait dans ce même cas, mais, sans que la première phase soit encore identifiable.

\section{REMONTAGE DE NUCLEUS PRISMATIQUES REPRIS POUR LA PRODUCTION D'ECLATS}

La reprise de nucléus prismatiques pour produire des éclats a pu être illustrée par le remontage d'une séquence de débitage provenant du niveau 10. II s'agit d'une reprise par un ravivage important du plan de frappe qui réduit la longueur de la table de débitage dans des proportions suffisantes pour passer d'une production de lames à une production d'éclats.

La séquence remontée comprend une série de lames et une série d'éclats, mais le nucléus n'a pas été retrouvé (Fig. 6). Le débitage se développe dans un gros galet de silex sénonien blond dont l'aménagement débute par la préparation d'un plan de frappe. Le débitage des lames est initié par le détachement d'une grosse lame semi-corticale sans qu'il y ait préparation de crête.

A la suite du débitage des lames, le plan de frappe a été ravivé (éclats de ravivage non retrouvés). La table de débitage, fortement raccourcie, n'a par la suite donné que des éclats, tous issus du nouveau plan de frappe. 


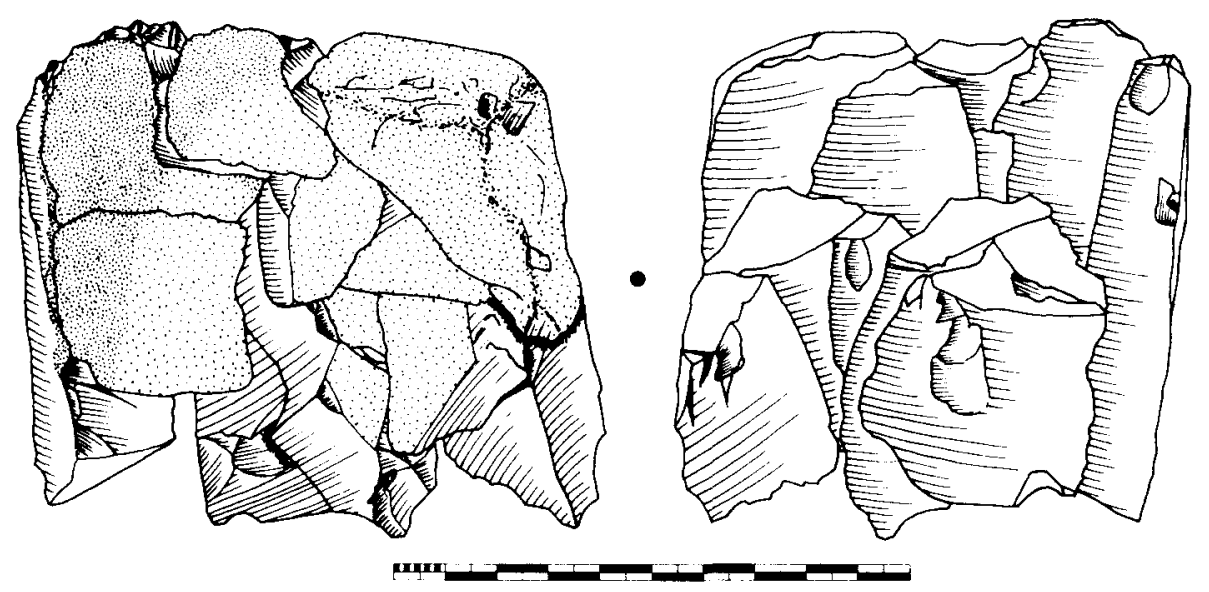

Fig. 6. Remontage d'un nucléus prismatique repris pour la production d'éclats du niveau 10 de l'abri Pataud (dessin L. Chiotti).

\section{CONCLUSION}

\section{1. Production de lames et d'éclats}

A la vue de la totalité du matériel des niveaux d'Aurignacien ancien de l'abri Pataud, il apparaît donc que la majeure partie des débitages effectués dans l'abri concernent la production d'éclats. En effet, en considérant l'ensemble de ces niveaux, nous n'avons pu dénombrer que 28 nucléus laminaires (en comptant ceux qui ont été repris pour donner des éclats), alors que les nucléus à éclats (tous types confondus) sont représentés par 143 pièces.

Même si on considère, comme cela a été évoqué précédemment, que certains nucléus à tendance prismatique ont pu être, dans un premier temps, des nucléus prismatiques à lames, la quantité de lames présentes dans l'Aurignacien ancien apparaît comme disproportionnée par rapport à la quantité de nucléus laminaires. Cela est d'autant plus net que la plupart du temps, les lames débitées dans l'abri le sont sur des blocs de silex disponibles à proximité immédiate, sous la forme de galets, qui sont généralement des blocs de petites dimensions n'ayant pu donner que relativement peu de lames (Chiotti, 1999). II y a donc une partie des lames qui n'a pas été produite sur le site, mais qui a été introduite déjà débitée.

Contrairement aux lames, la quasi-totalité des éclats ont été produits sur le site, le plus souvent à partir de galets de silex local, presque toujours de petites dimensions. Parmi les différentes techniques de production d'éclats décrites cidessus, nous pouvons distinguer deux grands groupes. 
- La technique la plus fréquente est celle du nucléus à tendance prismatique, consistant à préparer un ou plusieurs plans de frappe destinés à produire des éclats, souvent de forme allongée. Elle est représentée par 81 nucléus, soit plus de la moitié des nucléus à éclats de l'Aurignacien ancien.

II ne semble pas y avoir d'aménagement important du volume de ces blocs avant et au cours du débitage. Celui-ci est plutôt conduit de façon "opportuniste", en fonction des propriétés de chaque bloc.

- La seconde technique consiste à débiter des éclats sans préparation de plan de frappe. Elle regroupe principalement les nucléus globuleux, les nucléus informes et les débitages sur arête, soit un total de 48 pièces dans l'ensemble de l'Aurignacien ancien. Avec cette technique, il y a encore moins d'aménagement du volume puisqu'il n'y a plus aucune préparation de plan de frappe. Le débitage, d'une grande simplicité, exploite directement les surfaces qui se dégagent sur le bloc à la suite de chaque enlèvement.

Qu'il s'agisse de l'une ou l'autre de ces deux techniques, il y a donc très peu, voire pas d'aménagement du volume du bloc, ainsi qu'un certain opportunisme dans le déroulement du débitage. $F$. Bon qui décrit les industries aurignaciennes de la Grotte des Hyènes (Brassempouy, Landes) et du site de plein air de Régismont-le-Haut (Poilhes, Hérault) selon des modalités de débitage différentes fait cependant les mêmes remarques concernant l'utilisation relativement opportuniste des blocs, avec très peu d'aménagement du volume: "Ce n'est pas tant le volume général du bloc qui semble être important que certaines propriétés locales: angulation entre deux surfaces; dimensions relatives des surfaces" (Bon, 2002: 104).

\subsection{Morphologie et utilisation des éclats}

Lors des premières phases d'exploitation des blocs, les surfaces de débitages sont choisies de façon à produire des éclats de dimensions généralement comprises entre 4 et $6 \mathrm{~cm}$, rarement supérieures. Dans la majorité des cas, les nucléus retrouvés sont de petites dimensions, ce qui indique que le débitage se poursuit jusqu'à l'obtention d'éclats de dimensions assez réduites (inférieures à 2 $\mathrm{cm}$ ). Cela se confirme par l'étude morphométrique des éclats qui montre toujours une proportion importante de ces petits éclats (Chiotti, 1999).

D'autre part, cette étude nous a permis de montrer l'absence de recherche d'une morphologie d'éclat particulière lors de cette production. Pour tous les niveaux, les modules d'éclats sont assez variés, ce qui semble être le cas sur d'autres sites de la même période (Bon, 2002). La seule remarque que nous pouvons faire sur la forme des éclats est que les nucléus à tendance prismatique semblent produire des éclats un peu plus allongés. 
Les éclats ainsi produits ont été très fortement utilisés comme supports d'outils assez sommaires tels que les encoches, les denticulés, les pièces retouchées ou encore les pièces esquillées (Tableau V). Dans l'Aurignacien ancien de l'abri Pataud, les burins sont d' "assez mauvaise facture» et peuvent s'apparenter aux types précédemment cités, dans le sens où ils sont probablement réalisés rapidement pour un usage unique puis abandonnés. Ces burins sont également réalisés en majorité sur des éclats.

Tableau V. Répartition par supports de l'ensemble des outils de l'Aurignacien ancien de l'abri Pataud (niveaux 14 à 9).

\begin{tabular}{|c|c|c|c|c|}
\hline & Blocs & Éclats & Lames & Lamelles \\
\hline Grattoirs & & 79 & 249 & \\
\hline Grattoirs aurignaciens & 19 & 30 & 4 & \\
\hline Outils composites & & 6 & 26 & \\
\hline Perçoirs-becs & 1 & 32 & 33 & \\
\hline Burins & & 18 & 8 & \\
\hline Pièces tronquées & & 14 & 46 & \\
\hline Pièces à retouches continues sur un bord & & 65 & 142 & 9 \\
\hline Pièces à retouches continues sur deux bords & & 20 & 67 & \\
\hline Lames aurignaciennes & & & 30 & \\
\hline Pics & 17 & 5 & & \\
\hline Pièces à encoche & 1 & 271 & 103 & \\
\hline Pièces denticulée & & 48 & 14 & \\
\hline Pièces esquillée & & 63 & 36 & \\
\hline Racloirs & & 8 & & \\
\hline Outillage lamellaire & & & & 19 \\
\hline Divers & & 1 & & \\
\hline Pièces à retouches discontinues ou partielles & 1 & 301 & 133 & 4 \\
\hline Fragments d'outils & & 52 & 9 & \\
\hline
\end{tabular}

Quel que soit le niveau considéré, les éclats choisis comme support de l'outillage ne montrent pas de caractéristiques particulières qui pourraient traduire une sélection parmi l'ensemble de la production. La seule tendance semble être la recherche d'une certaine robustesse, avec des dimensions légèrement supérieures à la moyenne. 


\subsection{Comparaisons}

Comme nous l'avons vu, la plupart des études concernant le matériel lithique aurignacien qui présentent des données sur les nucléus montrent que la majorité des nucléus à éclats sont des nucléus globuleux ou informes (cf. supra).

Lors de la présente étude, nous avons distingué ce que nous avons nommé des nucléus à tendance prismatique. Il s'agit d'une catégorie de pièces que nous avons isolé du fait de leur technique qui nous paraît significativement différente de celle des nucléus globuleux et informes. Cependant, il ne s'agit certainement pas d'un type de débitage spécifique à l'Aurignacien ancien de l'abri Pataud. En effet, ce type de pièces semble avoir été classé par les différents auteurs parmi les nucléus globuleux et/ou informes, ce qui est certainement lié au fait que lorsque l'exploitation des blocs est poussée, les nucléus résultant des deux techniques présentent des formes assez proches. Ce classement dans une même catégorie apparaît d'autant plus évident si l'on tient compte de la remarque de A. Cheynier qui précise que «les nucléus globuleux présentent parfois un semblant de plan de frappe irrégulier" (Cheynier 1949: 51).

En définitive, et malgré la présence de techniques différentes, la production d'éclats de l'Aurignacien et en particulier de l'Aurignacien ancien apparaît toujours comme relativement opportuniste, avec une exploitation maximale des différentes surfaces des blocs. Cela est très net pour les débitages globuleux et informes pour lesquels il n'y a pas de surface de débitage privilégiée, mais c'est également le cas pour les nucléus à tendance prismatique, en particulier lorsqu'il y a plusieurs plans de frappe qui en règle générale n'ont aucun rapport entre eux (surfaces de débitage différentes et sans orientations particulières sur le bloc).

Cet aspect opportuniste rejoint en quelques sortes l'analyse que fait $F$. Bon des débitages aurignaciens. En effet, même si cette analyse est différente, avec l'identification de deux types de débitages (trifacial et parallélépipédique), l'aspect principal semble être que le débitage est fortement tributaire de la morphologie des supports sur lesquels ils sont effectués ainsi que des propriétés locales des blocs (Bon, 1996, 2002), ce qui dénote donc également d'un certain opportunisme.

\section{BIBLIOGRAPHIE}

BOËDA, E. (1993): Le débitage discoïde et le débitage Levallois récurrent centripète. Bulletin de la Société Préhistorique Française, t. 90, 6: 392-404

- (1994): Le concept Levallois: variabilité des méthodes. Paris, éditions du CNRS (Monographies du CRA, 9), 280 pag.

BON, F. (1996): L'industrie lithique aurignacienne de la couche $2 \mathrm{~A}$ de la grotte des Hyènes à Brassempouy (Landes), in Pyrénées Préhistoriques Arts et Sociétés, éditions. du CTHS, actes du 118ème Congrès National des Sociétés Historiques et Scientifiques, Pau, 1993: 439-455.

- (2002): L'Aurignacien entre mer et océan. Réflexion sur l'unité des phases anciennes de l'Aurignacien dans le sud de la France. Paris, Société Préhistorique Française (Mémoire de la Société Préhistorique Française, $X X \mid X), 253$ pag. 
BON, F., Bodu, P. (2002): Analyse technologique du débitage aurignacien, in L'Aurignacien de la grotte du Renne. Les fouilles d'André Leroi-Gourhan à Arcy-sur-Cure (Yonne), Paris, éditions du CNRS (Supplément à Gallia Préhistoire, XXXIV): 115-133.

Bordes, F. (1950): Principes d'une méthodologie d'étude des techniques et de la typologie du Paléolithique ancien et moyen, l'Anthropologie, t. 54: 19-34.

BreZILLON, M. N. (1968): La dénomination des objets de pierre taillée. Matériaux pour un vocabulaire des préhistoriens de langue française. Paris, éditions du CNRS (Supplément à Gallia Préhistoire, IV), 411 pag.

CHEYNIER, A. (1949): Badegoule, station solutréenne et Proto-magdalénienne. Paris, (Archives de l'I.P.H., 23), 230 pag.

CHIOTI, L. (1999): Les industries lithiques des niveaux aurignaciens de l'abri Pataud, Les Eyzies-deTayac (Dordogne): étude technologique et typologique, thèse de Doctorat du Muséum national d'histoire naturelle, Paris, 2 tomes, 839 pag.

Chiotti, L.; Patou-Mathis, M. el Vercoutere, C. (2003): Comportements techniques et de subsistance à l'Aurignacien ancien: la couche 11 de l'abri Pataud, (Dordogne), Gallia Préhistoire, 45.

Demars, P.-Y. (1994): L'économie du silex au Paléolithique supérieur dans le nord de l'Aquitaine. Analyse, synthèse et interprétations, thèse de doctorat d'Etat de l'Université de Bordeaux 1, 2 volumes, 549 pag. et 270 pag.

GuichARD, J. et G., MORALA, A. (1989): Rémanence de la technique Levallois au Paléolithique supérieur ancien. Deux sites du Bergeracois: Canaule I et Troche (Creysse), Documents d'Archéologie périgourdine, ADRAP, t. 4: 5-20.

InizAn, M.-L.; Reduron, M.; Roche, H. et TIXIER, J. (1995): Technologie de la pierre taillée. Meudon, éditions du cercle de recherches et d'études préhistoriques (Préhistoire de la pierre taillée, 4), 199 pag.

LAPLACE, G. (1964): Essai de typologie systématique. Annali dell Universata di Ferrara, sezione 15, suppl. 2 al vol. I, 85 pag.

Lerol-Gourhan, A. (1964): Notes de morphologie descriptive. Cours de Préhistoire. Paris, F.L.S.H., 33 pag.

Movius, H. L. Jr. (1977): Excavation of the abri Pataud, Les Eyzies (Dordogne): Stratigraphy. Harvard University, Cambridge, Massachussetts, Peabody Museum (American School of Prehistoric Research, 31), 167 pag., plans séparés.

Perpere, M. et Schmider, B. (2002): Les nucléus et les produits de débitage. Données numériques et morphologiques, in L'Aurignacien de la grotte du Renne. Les fouilles d'André Leroi-Gourhan à Arcysur-Cure (Yonne), Paris, éditions du CNRS (Supplément à Gallia Préhistoire, XXXIV): 135-141.

SONNEVILLE-Bordes, D. de (1960): Le Paléolithique supérieur en Périgord. Bordeaux, Delmas, 2 vol., 560 pag.

- (1970): Les industries aurignaciennes de l'abri Caminade-Est, commune de La Canéda (Dordogne), Quaternaria, XIII: 77-131.

TiXIER, J. (1960): Les industries lithiques d'Aïn Fritissa (Maroc oriental), Bulletin d'archéologie marocaine, t. III: 107-244.

Tixier J.; InIZAN, M.-L.; Roche, H. et Dauvois, M. (1980): Terminologie et technologie. Antibes, éditions du cercle de recherches et d'études préhistoriques (Préhistoire de la pierre taillée, 1), 120 pag. 